\title{
Phase I study of daily and weekly regimens of the orally administered MDM2 antagonist idasanutlin in patients with advanced tumors
}

\author{
Antoine Italiano ${ }^{1,2,3}$. Wilson H. Miller Jr ${ }^{4}$. Jean-Yves Blay ${ }^{5}$. Jourik A. Gietema ${ }^{6}$. Yung-Jue Bang ${ }^{7}$. \\ Linda R. Mileshkin ${ }^{8} \cdot$ Hal W. Hirte $^{9} \cdot$ Brian Higgins $^{10} \cdot$ Steven Blotner ${ }^{10} \cdot$ Gwen L. Nichols $^{10} \cdot$ Lin Chi Chen $^{10}$. \\ Claire Petry ${ }^{11}$. Qi Joy Yang ${ }^{12}$. Christophe Schmitt ${ }^{11}$. Candice Jamois ${ }^{11}$ • Lillian L. Siu ${ }^{13}$
}

Received: 22 March 2021 / Accepted: 15 June 2021 / Published online: 28 June 2021

(C) The Author(s) 2021

\begin{abstract}
Summary Aim The oral MDM2 antagonist idasanutlin inhibits the p53-MDM2 interaction, enabling p53 activation, tumor growth inhibition, and increased survival in xenograft models. Methods We conducted a Phase I study of idasanutlin (microprecipitate bulk powder formulation) to determine the maximum tolerated dose (MTD), safety, pharmacokinetics, pharmacodynamics, food effect, and clinical activity in patients with advanced malignancies. Schedules investigated were once weekly for 3 weeks $(\mathrm{QW} \times 3)$, once daily for 3 days $(\mathrm{QD} \times 3)$, or $\mathrm{QD} \times 5$ every 28 days. We also analyzed $\mathrm{p} 53$ activation and the anti-proliferative effects of idasanutlin. Results The dose-escalation phase included 85 patients $(\mathrm{QW} \times 3, \mathrm{n}=36$; $\mathrm{QD} \times 3$, $\mathrm{n}=15$; $\mathrm{QD} \times 5, \mathrm{n}=34)$. Daily MTD was $3200 \mathrm{mg}(\mathrm{QW} \times 3), 1000 \mathrm{mg}(\mathrm{QD} \times 3)$, and $500 \mathrm{mg}(\mathrm{QD} \times 5)$. Most common adverse events were diarrhea, nausea/vomiting, decreased appetite, and thrombocytopenia. Dose-limiting toxicities were nausea/ vomiting and myelosuppression; myelosuppression was more frequent with QD dosing and associated with pharmacokinetic exposure. Idasanutlin exposure was approximately dose proportional at low doses, but less than dose proportional at $>600$ $\mathrm{mg}$. Although inter-patient variability in exposure was high with all regimens, cumulative idasanutlin exposure over the whole 28-day cycle was greatest with a $\mathrm{QD} \times 5$ regimen. No major food effect on pharmacokinetic exposure occurred. MIC-1 levels were higher with QD dosing, increasing in an exposure-dependent manner. Best response was stable disease in 30.6\% of patients, prolonged ( $>600$ days) in 2 patients with sarcoma. Conclusions Idasanutlin demonstrated dose- and scheduledependent p53 activation with durable disease stabilization in some patients. Based on these findings, the QD $\times 5$ schedule was selected for further development.
\end{abstract}

Trial registration NCT01462175 (ClinicalTrials.gov), October 31, 2011.

Keywords p53 activation $\cdot$ MDM2 antagonist $\cdot$ Cis-imidazoline analog $\cdot$ Idasanutlin $\cdot$ Nutlin $\cdot$ MIC-1

Lillian L. Siu

lillian.siu@uhn.ca

Institut Bergonié, Bordeaux, France

Gustave Roussy, Villejuif, France

3 Faculty of Medicine, University of Bordeaux, Bordeaux, France

4 Segal Cancer Centre, Jewish General Hospital, McGill University, Montreal, QC, Canada

5 Centre Léon Bérard, Lyon, France

6 University Medical Center Groningen, University of Groningen, Groningen, the Netherlands
7 Seoul National University College of Medicine, Seoul, South Korea

8 Peter MacCallum Cancer Center, Melbourne, Australia

9 Juravinski Cancer Centre, Hamilton, ON, Canada

10 Roche Innovation Center, Hoffmann-La Roche, New York, NY, USA

11 Roche Innovation Center, Basel, Switzerland

12 Certara, Montréal, QC, Canada

13 Princess Margaret Cancer Centre, Toronto, ON, Canada 


\section{Introduction}

The p53 tumor suppressor protein is a powerful pro-apoptotic factor that plays a central role in inhibiting tumor development [1]. MDM2, a negative regulator of the p53 tumor suppressor, functions as an E3 ubiquitin ligase, targeting p53 for proteasomal degradation [2]. A class of imidazoline compounds, termed "Nutlins," has been identified as potent and selective inhibitors of the p53-MDM2 interaction, interacting specifically with the p53 binding pocket of MDM2 and thus releasing p53 from negative control [3-5]. Using Nutlins to treat cancer cells that express functional p53 stabilizes p53 and activates its pathway, leading to activation of p53-target genes, cell cycle arrest, apoptosis, and/or senescence [4]. RG7112, the first Nutlin compound to enter the clinic, established clinical proof of mechanism in MDM2-amplified liposarcoma [6, 7]. Further, response per Response Evaluation Criteria in Solid Tumors (RECIST) was achieved in previous RG7112 clinical trials. However, patients had difficulty tolerating treatment on the requisite daily RG7112 schedule [6, 8].

Idasanutlin (RG7388, RO5503781) is a secondgeneration selective inhibitor of the p53-MDM2 interaction. Idasanutlin retains the in vitro anti-tumor activity of RG7112 but has superior pharmacologic characteristics, including improved potency, bioavailability, and selectivity for the p53 binding site of MDM2 [5, 9-15]. In preclinical modeling and simulation studies, both daily and intermittent dosing with idasanutlin were predicted to be effective at achieving tumor stasis $[10,11]$. Thus, despite the relatively short half-lives of p53 and MDM2, continuous suppression of the p53-MDM2 interaction did not appear to be required for optimal antitumor activity. These preclinical data suggested that intermittent idasanutlin dosing could provide the same activity as daily dosing.

The preclinical evidence together with the improved pharmacological properties of idasanutlin supported the rationale for shorter intermittent dosing schedules in this initial Phase I study, with the aim of decreasing the risk of thrombocytopenia. The aim of this first-in-human study was to investigate the maximum tolerated dose (MTD), characterize dose-limiting toxicity (DLT), and explore the safety, tolerability, pharmacokinetics (PK), pharmacodynamics (PD), and clinical responses with idasanutlin with different dosing schedules.

\section{Materials and methods}

\section{Study design and treatment}

This was a multicenter, open-label, Phase I, multiple ascending dose-escalation trial of single-agent idasanutlin in a microprecipitate bulk powder (MBP) formulation in patients with advanced malignancies other than leukemia (ClinicalTrials. gov identifier: NCT01462175). Following the accelerated dose-escalation phase using an escalation with overdose control (EWOC) design [16], additional patients were recruited to food-effect and apoptosis-imaging cohorts (only safety data reported; Fig. 1). The dose-escalation phase involved singlepatient cohorts until grade 2 related adverse events (AE) were reported (Fig. 1). Based on these AE criteria, subsequent dose escalation involved 3-patient cohorts in a modified continualreassessment-method EWOC design. Idasanutlin was administered either once daily (QD; (to define abbreviation at first use of term) when total daily dose was at or below $800 \mathrm{mg}$ ) or twice daily (when total daily dose was at or exceeded $800 \mathrm{mg}$ ). For the weekly regimen, idasanutlin was administered orally at a total daily dose of 100-3200 mg once weekly (QW) for 3 weeks $(\mathrm{QW} \times 3)$ followed by 13 days of rest. For the daily regimens, idasanutlin was administered orally at a total daily dose of $1000 \mathrm{mg}$ or $1600 \mathrm{mg}$ QD for 3 days $(\mathrm{QD} \times 3$ ) followed by 25 days of rest, or at a total daily dose of $100-1200 \mathrm{mg}$ daily for 5 days $(\mathrm{QD} \times 5)$ followed by 23 days of rest. Idasanutlin was given without food, except in the food effect sub-study. Treatment continued until disease progression, unacceptable toxicity, or withdrawal of consent.

\section{Patients}

Eligible patients were at least 18 years of age with histologically confirmed advanced malignancies (except any type of leukemia) for which standard curative or palliative measures were unavailable, no longer effective, or unacceptable to the patient. All patients were required to have measurable disease per RECIST 1.1 criteria (or Cheson criteria for malignant lymphomas) and Eastern Cooperative Oncology Group performance status of 0 to 1. There were no limitations on additional type or amount of prior anti-tumor therapy. Acute toxicities from any prior anti-cancer therapy, surgery, or radiotherapy must have resolved to grade $\leq 1$ per National Cancer Institute Common Terminology Criteria for Adverse Events v4.03. Patients had to have adequate bone marrow function, renal function (serum creatinine within normal limits or creatinine clearance $\geq 50 \mathrm{~mL} / \mathrm{min}$ [calculated by CockcroftGault equation]), and hepatic function (with serum total bilirubin $\leq 2 \mathrm{mg} / \mathrm{dL}$ ). Those with stable central nervous system metastases or chronic, stable, and rate-controlled atrial fibrillation were eligible. To be eligible for the biomarker cohort, patients had to have a tumor that could be biopsied prior to and during treatment. Exclusion criteria included use of other investigational drugs $\leq 4$ weeks prior to study treatment start; preexisting gastrointestinal disorders that could interfere with drug absorption; and history of seizure disorders or unstable central nervous system metastases. 
Fig. 1 Study design and treatment allocation schedules. A Study overview. B Treatment allocation for $\mathrm{QW} \times 3$ schedule (schedule A). C Treatment allocation for $\mathrm{QD} \times 3$ and $\mathrm{QD} \times 5$ schedules (schedule $\mathrm{B}$ ). For the weekly regimen (schedule A), idasanutlin was administered orally at a total daily dose of 100-3200 mg once weekly for 3 weeks followed by 13 days of rest. For the daily regimens (schedule B), idasanutlin was administered orally at a total daily dose of $1000 \mathrm{mg}$ or $1600 \mathrm{mg}$ daily for 3 days followed by 25 days of rest, or at a total daily dose of 100-1200 mg daily for 5 days followed by 23 days of rest. Arrows indicate the sequence of opening the cohorts (panels $\mathbf{B}$ and $\mathbf{C}$ ). Gray boxes indicate cohorts with 3 planned dosing days per cycle only. AE, adverse event; BID, twice daily; EWOC, escalation with overdose control; QD, once daily; QW, once weekly. ${ }^{a}$ Not all EWOC cohorts had associated biomarker evaluations. ${ }^{\mathrm{b}}$ One patient was dosed BID. ${ }^{c}$ Patients were dosed BID
A.

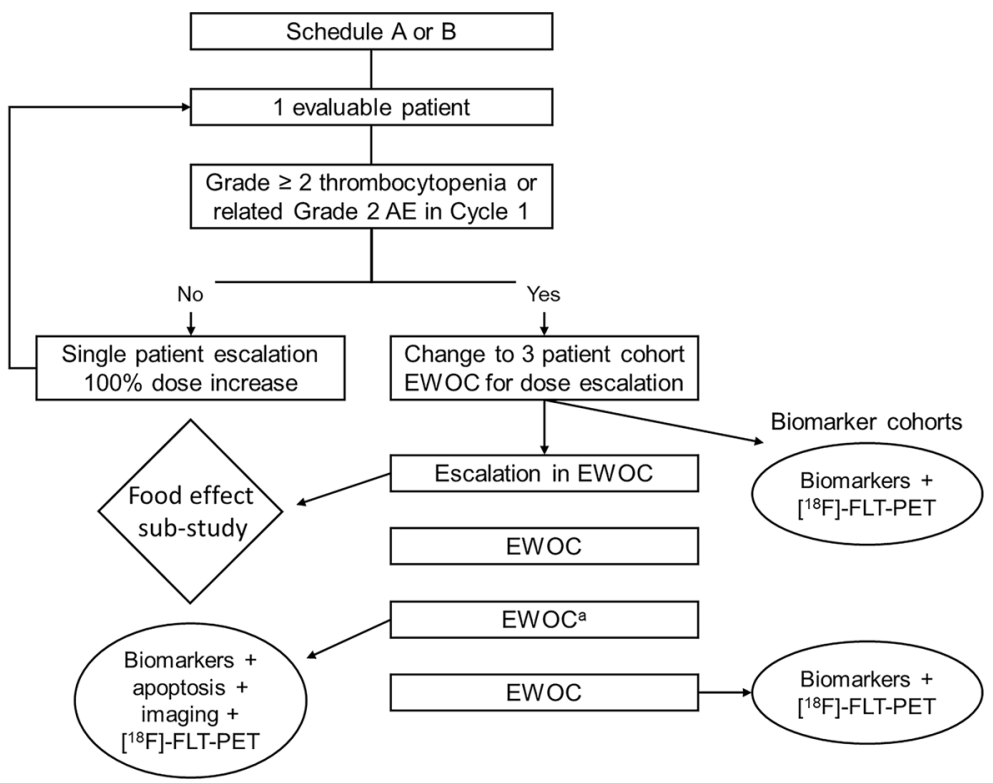

B.

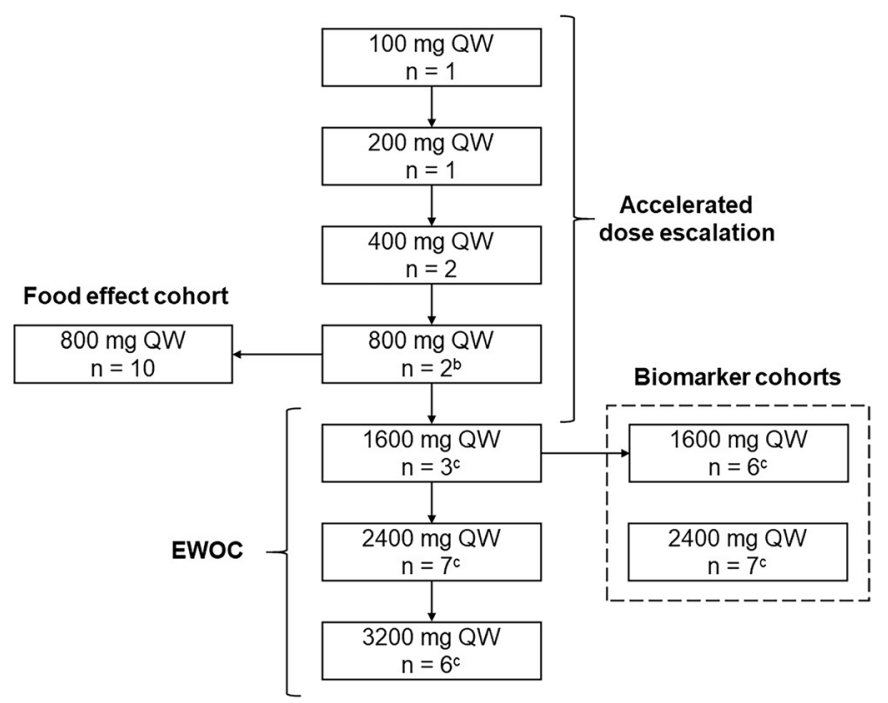

C.

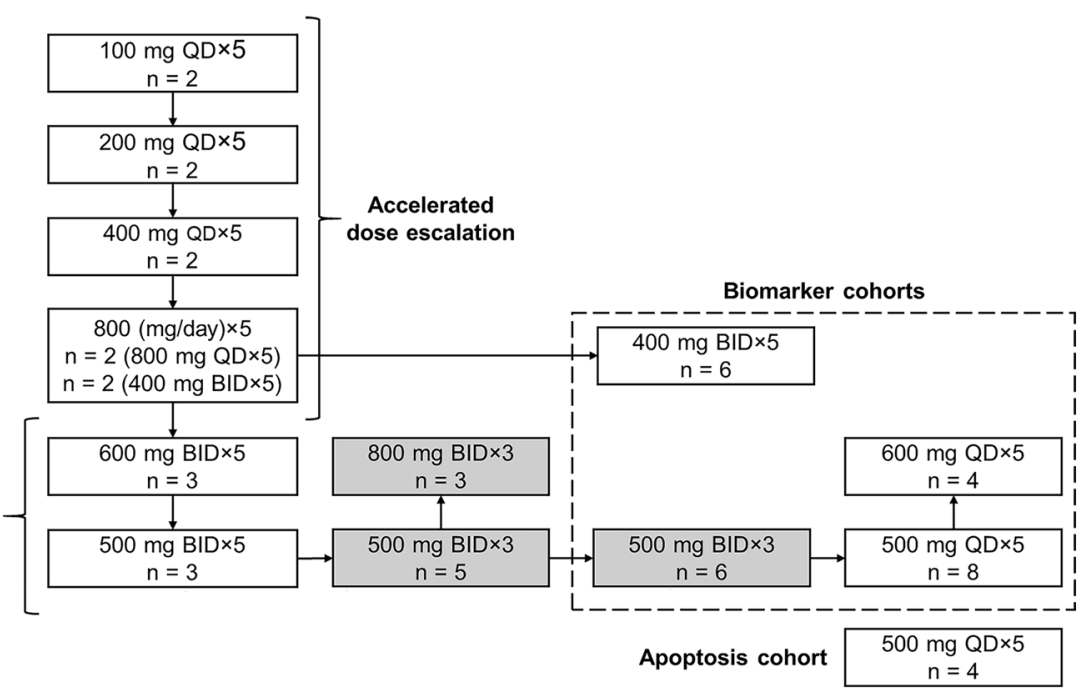




\section{Endpoints and assessments}

The primary study objectives were to: determine the MTD of different idasanutlin dosing schedules administered in 28-day cycles to fasted patients; characterize the DLTs and overall safety profile across escalated dose levels; and explore these dosing schedules for safety and tolerability. Secondary objectives included determining the PK and PD profiles of idasanutlin, as well as clinical responses.

The safety population, defined as all patients who received $\geq 1$ dose of the study drug, was used for all safety, efficacy, and PK analyses. The DLT-evaluable population comprised patients who completed the first 28-day treatment cycle (i.e., received $\geq 4$ days of therapy in the $\mathrm{QD} \times 5$ schedule or $\geq 2$ days in the $\mathrm{QW} \times 3$ schedule) and had sufficient safety evaluations. AEs were reported according to the Medical Dictionary for Regulatory Activities, version 17.0, with severity graded by National Cancer Institute Common Terminology Criteria for Adverse Events v4.03.

Efficacy-evaluable patients included those in the safety population with a baseline assessment and $\geq 1$ post-baseline tumor assessment. Tumor response (objective response rate) was assessed approximately every 8 weeks using RECIST 1.1 criteria for patients with measurable or non-measurable lesions or using Cheson criteria for those with malignant lymphomas.

Further details on the assessments of the food effect, PK (Online Resource Table S1), PD, and biomarkers are available in the Supplementary Methods in the Online Resource.

\section{Results}

\section{Patient population}

A total of 99 patients were enrolled and treated between November 15, 2011, and July 4, 2014. Dose-escalation cohorts included those on the QW $\times 3(n=36), Q D \times 3$ $(\mathrm{n}=15)$, and $\mathrm{QD} \times 5(\mathrm{n}=34)$ regimens; 10 and 4 patients were included in the food-effect and apoptosis-imaging cohorts, respectively (Fig. 1). Thirty-seven patients from the dose-escalation phase were included in the biomarker analysis cohorts $(\mathrm{QW} \times 3, \mathrm{n}=13$; $\mathrm{QD}$ regimens, $\mathrm{n}=24)$.

Baseline characteristics were similar across cohorts; the median age was 57.0 years (range, 23-76 years; Online Resource Table S2). More than two-thirds of patients (69.4\%) had an Eastern Cooperative Oncology Group performance status of 1 . All patients had been treated with $\geq 1$ prior anti-cancer regimen, with the majority $(70.7 \%)$ having received 1 to 4 regimens. The most common tumor types were sarcoma $(n=23[23.2 \%])$ and colorectal cancer $(\mathrm{n}=12[12.1 \%])$. TP53 mutations were analyzed at baseline (archival and pre-dose tumor samples) and, where possible, in paired pre- and post-biopsy samples. Mutations in TP53 were detected in 19 of 58 archival samples $(32.8 \%)$ and 12 of 32 pre-dose biopsies (37.5\%) (Online Resource Table S2).

\section{Safety and tolerability}

The median duration of treatment for all patients was 36 days (range, $1-726$ days), with 15 patients (15.2\%) treated for $>91$ days (Online Resource Table S3). The median (range) number of total daily doses received in the $\mathrm{QW} \times 3, \mathrm{QD} \times 3$, and $\mathrm{QD} \times 5$ cohorts, respectively, was 10.5 (2-72), 9.0 (6-42), and 10.0 (1-130). All 99 patients comprised the safety population; across all cohorts, 78 patients (78.8\%) received $\leq 2$ treatment cycles.

The MTD for QW $\times 3$ dosing was $3200 \mathrm{mg}$ (given as $1600 \mathrm{mg}$ twice daily [BID]), with DLTs of nausea, thrombocytopenia, and vomiting (Online Resource Table S4), all reported at a total daily dose of $1600 \mathrm{mg}$ or higher. The MTD for QD $\times 3$ dosing was $1000 \mathrm{mg}$ (given as $500 \mathrm{mg}$ BID), with DLTs of thrombocytopenia, febrile neutropenia, neutropenia, and pancytopenia. For QD $\times 5$ dosing, the MTD was $500 \mathrm{mg}$ (given QD), with DLTs of thrombocytopenia, neutropenia, febrile neutropenia, and diarrhea. A total of 31 DLTs were reported across all cohorts $(\mathrm{n}=99)$, with 21 patients $(21.2 \%$; dose-escalation cohorts, $\mathrm{n}=20$; apoptosis cohort, $\mathrm{n}=1$ ) having $\geq 1$ DLT. The most common DLT was thrombocytopenia, occurring in 16 of 99 patients (16.2\%). Other DLTs included neutropenia (5 [5.1\%]), febrile neutropenia (3 [3.0\%]), nausea (2 [2.0\%]), as well as leukopenia, pancytopenia, diarrhea, and vomiting (1 each [1.0\%]). Within the dose-escalation cohorts, DLTs were more common in patients on daily $(40 \%$ for $\mathrm{QD} \times 3$ and $32.4 \%$ for $\mathrm{QD} \times 5$ ) versus weekly dosing schedules $(8.3 \%)$, with a higher incidence of DLTs related to hematologic and lymphatic system disorders reported with daily regimens (Online Resource Table S4).

All 99 patients experienced $\geq 1 \mathrm{AE}$ that was considered by the investigator to be related to study treatment (Table 1). The most common treatment-related AEs were diarrhea (74.7\%), nausea $(71.7 \%)$, vomiting $(50.5 \%)$, decreased appetite (43.4\%), and thrombocytopenia (39.4\%; Online Resource Table S5). In general, treatment-related AEs occurred at the highest frequencies with the $\mathrm{QD} \times 3$ schedule; the lowest frequencies were observed with the $\mathrm{QD} \times 5$ schedule (Online Resource Table S5).

Grade $\geq 3$ AEs of any cause occurred in 63 patients (63.6\%) and were reported in higher incidences in the QD dosing regimens (Table 1). The most common grade $\geq 3$ anycause AEs were thrombocytopenia (29.3\%), anemia (20.2\%), neutropenia (16.2\%), nausea (11.1\%), and diarrhea (7.1\%). Serious AEs (SAEs) were reported in 32 patients (32.3\%) across all study groups (Table 1; Online Resource Table S6). 
Table 1 Overview of AEs

\begin{tabular}{|c|c|c|c|c|c|c|}
\hline & \multirow{2}{*}{$\begin{array}{l}\text { Weekly dos- } \\
\text { ing }(\mathrm{QW} \times 3)^{\mathrm{a}} \\
(\mathrm{n}=36)\end{array}$} & \multicolumn{2}{|c|}{ Daily dosing ${ }^{\mathrm{b}}$} & \multirow{2}{*}{$\begin{array}{l}\text { Food effect cohort } \\
(\mathrm{n}=10)\end{array}$} & \multirow{2}{*}{$\begin{array}{l}\text { Apoptosis cohort } \\
(\mathrm{n}=4)\end{array}$} & \multirow{2}{*}{$\begin{array}{l}\text { Total } \\
(\mathrm{N}=99)\end{array}$} \\
\hline & & $\begin{array}{l}\mathrm{QD} \times 3 \\
(\mathrm{n}=15)\end{array}$ & $\begin{array}{l}\mathrm{QD} \times 5 \\
(\mathrm{n}=34)\end{array}$ & & & \\
\hline \multicolumn{7}{|l|}{ Total no. of events } \\
\hline SAEs, $\mathrm{n}$ & 10 & 12 & 27 & 2 & 2 & 53 \\
\hline DLT AEs, $\mathrm{n}$ & 4 & 8 & 17 & 0 & 2 & 31 \\
\hline Deaths (due to fatal AE or PD), n (\%) & $2(5.6)$ & 0 & $4(11.8)$ & $1(10.0)$ & 0 & $7(7.1)$ \\
\hline \multicolumn{7}{|l|}{ No. of patients with $\geq 1$ event, $\mathrm{n}(\%)$} \\
\hline $\mathrm{AE}$ & $36(100.0)$ & $15(100.0)$ & $34(100.0)$ & $10(100.0)$ & $4(100.0)$ & $99(100.0)$ \\
\hline Related AE & $36(100.0)$ & $15(100.0)$ & $34(100.0)$ & $10(100.0)$ & $4(100.0)$ & $99(100.0)$ \\
\hline Grade $\geq 3 \mathrm{AE}$ & $21(58.3)$ & $11(73.5)$ & $25(73.5)$ & $4(40.0)$ & $2(50.0)$ & $63(63.6)$ \\
\hline SAE & $9(25.0)$ & $7(46.7)$ & $13(38.2)$ & $2(20.0)$ & $1(25.0)$ & $32(32.3)$ \\
\hline Related SAE & $4(11.1)$ & $7(46.7)$ & $13(38.2)$ & 0 & $1(25.0)$ & $25(25.3)$ \\
\hline DLT AE & $3(8.3)$ & $6(40.0)$ & $11(32.4)$ & 0 & $1(25.0)$ & $21(21.2)$ \\
\hline AE with fatal outcome & $1(2.8)$ & 0 & $1(2.9)$ & 0 & 0 & $2(20.0)$ \\
\hline AE leading to treatment withdrawal & $4(11.1)$ & $3(20.0)$ & $10(29.4)$ & $1(10.0)$ & 0 & $18(18.2)$ \\
\hline AE leading to dose modification/interruption & $16(44.4)$ & $7(46.7)$ & $18(52.9)$ & $2(20.0)$ & $1(25.0)$ & $44(44.4)$ \\
\hline
\end{tabular}

$A E$ adverse event, $D L T$ dose-limiting toxicity, $P D$ progressive disease, $Q D$ once daily, $Q W$ once weekly, $S A E$ serious adverse event

${ }^{a}$ Weekly dosing schedule, including biomarker cohorts, except those enrolled in the food effect sub-study

${ }^{\mathrm{b}}$ Daily dosing schedules $(\mathrm{QD} \times 3$ or $\mathrm{QD} \times 5)$, including biomarker cohorts, except those enrolled in the apoptosis cohort

Treatment-related SAEs were reported in 25 patients (25.3\%); the most frequently reported (in 24 of 25 patients) were related to blood and lymphatic system disorders: thrombocytopenia/decreased platelet count (14 events), febrile neutropenia (5 events), neutropenia/decreased neutrophil count (4 events), leukopenia/decreased white blood cell count (3 events), and anemia (2 events). Treatment-related SAEs were more frequently reported with QD dosing (QD $\times 3,7$ of 15 [46.7\%]; QD $\times 5,13$ of 34 [38.2\%]) than $\mathrm{QW} \times 3$ dosing (4 of 36 [11.1\%]) (Online Resource Table S6).

The majority of patients (81 of 99 [81.8\%]) discontinued treatment due to non-safety reasons: disease progression $(\mathrm{n}=77)$, patient consent withdrawal $(\mathrm{n}=3)$, and other reason unspecified $(\mathrm{n}=1)$. Eighteen patients $(18.2 \%)$ withdrew due to AEs, 8 of which were considered SAEs. More AE-related discontinuations occurred in patients receiving daily dosing regimens, excluding the apoptosis imaging cohort $(\mathrm{QD} \times 3$, $20.0 \%$; QD $\times 5,29.4 \%$ ), compared with those receiving $\mathrm{QW} \times 3$, excluding the food effect cohort (11.1\%). The most common AEs resulting in study drug discontinuation among all patients were neutropenia, thrombocytopenia, and pulmonary embolism (3.0\% each). AEs associated with study withdrawal were more likely to be hematologic in nature and grade $\geq 3$ in severity.

Dose modifications/interruptions due to an $\mathrm{AE}$ were reported in 44 patients (44.4\%) and occurred at similar frequencies with the weekly and daily schedules (Table 1). This included 16 of 36 patients (44.4\%) on $\mathrm{QW} \times 3$ dosing, while 7 of 15 patients $(46.7 \%)$ on QD $\times 3$ dosing and 18 of 34
(52.9\%) on $\mathrm{QD} \times 5$ dosing required dose modifications. The most frequently reported AEs leading to dose modification were thrombocytopenia (24.2\%) and neutropenia (9.1\%).

Overall, 7 deaths occurred during treatment or over the 28 days following the last study dose: 5 were due to progressive disease and 2 were due to an SAE (Table 1). One death $(\mathrm{QW} \times 3$ cohort $)$ was due to an intra-abdominal hemorrhage and pulmonary embolism, both determined to be unrelated to study treatment. The other death $(\mathrm{QD} \times 5$ cohort $)$ was due to pulmonary embolism and possibly related to study treatment.

\section{Clinical activity}

Of the 85 response-evaluable patients, none achieved an objective response, and 26 patients (30.6\%) had a best overall response of stable disease (SD). Of the 26 patients with SD, 7 had modest tumor shrinkage not approaching objective response $(-1,-4,-7,-8,-8,-9$, and $-11 \%)$. Rates of SD were comparable among patients receiving weekly dosing (10 of 33 patients; $30.3 \%)$ and daily dosing (QD $\times 3,5$ of 13 [38.5\%]; QD $\times 5,8$ of 26 [30.8\%]). Median overall duration of SD was 72.5 days (range, 8-696 days); this was shorter in the $\mathrm{QD} \times 3$ cohort (57.0 days) than in the $\mathrm{QW} \times 3$ or $\mathrm{QD} \times 5$ cohorts ( 99.0 and 103.0 days, respectively). For patients who were treated $>3$ cycles, the duration of idasanutlin exposure is shown in Online Resource Fig. S1.

Ten patients ( 7 with sarcoma, 1 with melanoma, 1 with testicular cancer, and 1 with mesothelioma) experienced SD 
A.

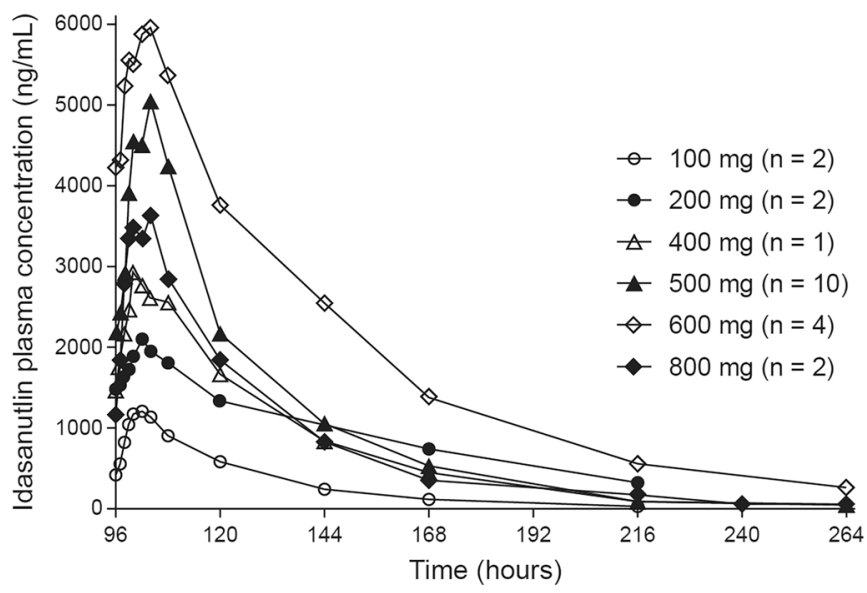

B.

First dose (Day 1)

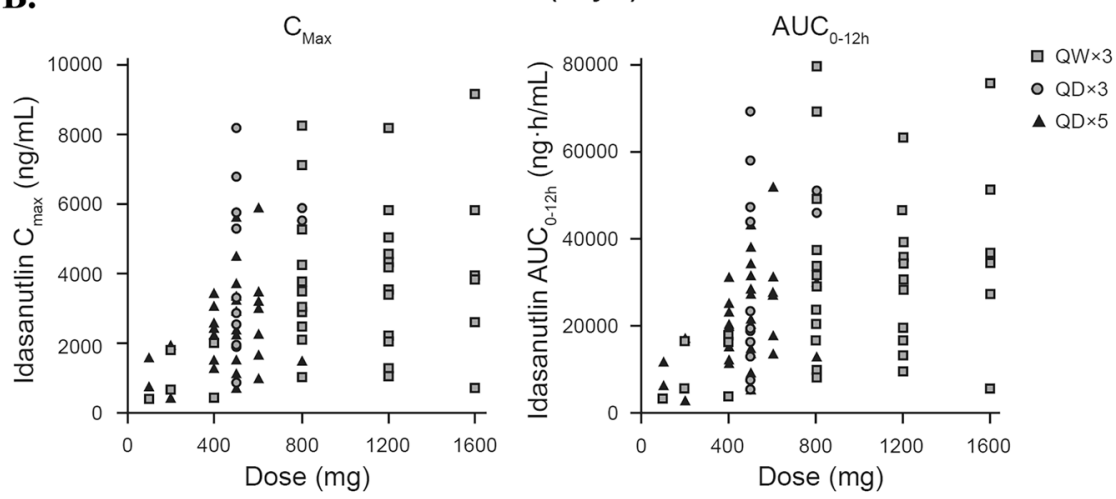

Last dosing day (day 3 for $Q D \times 3$ or day 5 for $Q D \times 5$ )
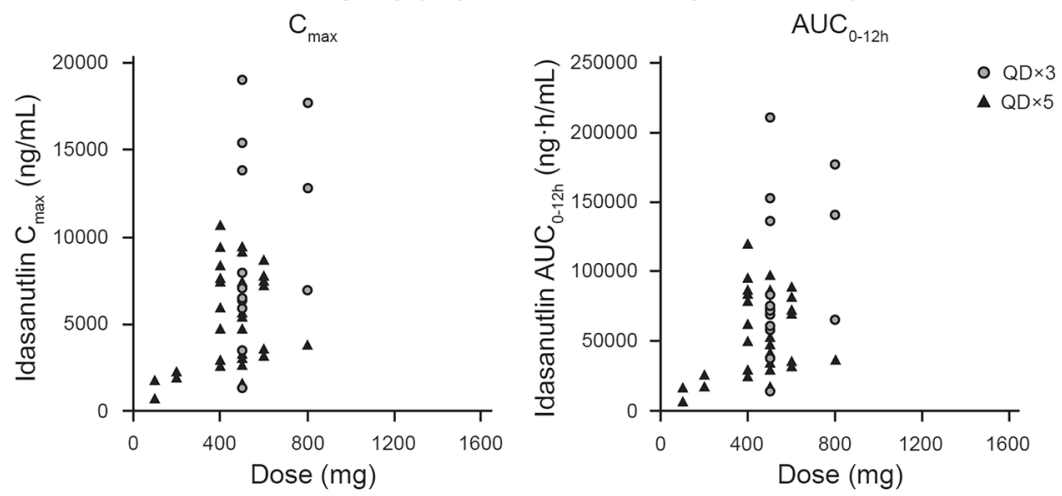

C.

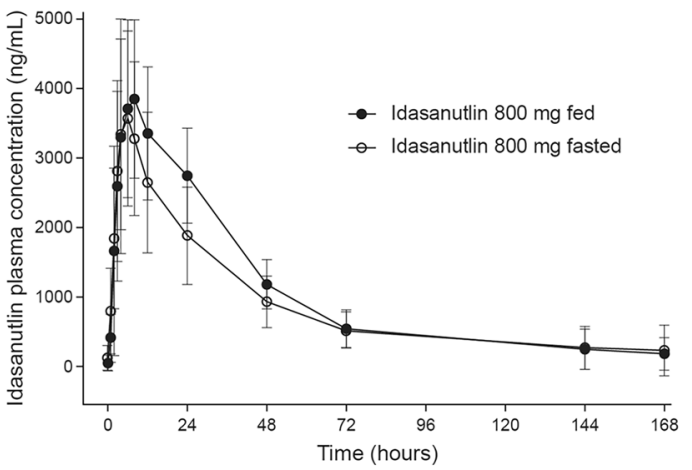

D.

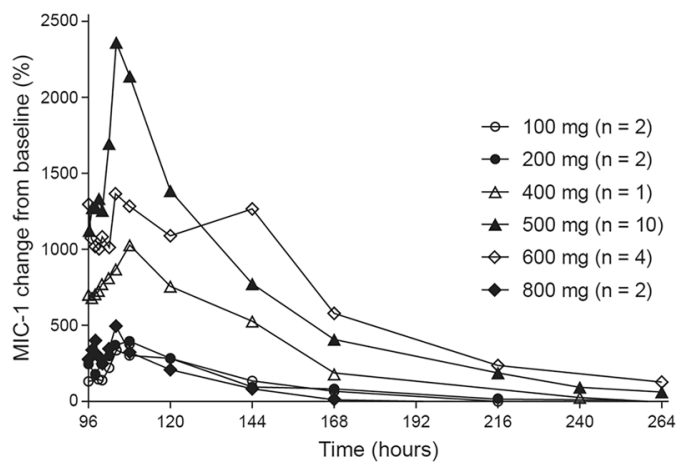


४Fig. 2 Idasanutlin pharmacokinetics and pharmacodynamics. A Mean plasma concentration-time profiles of idasanutlin following oral administration of once daily dose for 5 days; data for last dose on day 5 (dose escalation, biomarker, and apoptosis cohort). B Scatterplots of idasanutlin exposure versus administered dose on first and last days of idasanutlin treatment. Data for last dosing day do not include patients who received $\mathrm{QW} \times 3$ dosing. C Plasma concentration-time profiles of idasanutlin following oral administration of a single 800$\mathrm{mg}$ dose in the fasted and fed state. Data are presented as arithmetic mean values $(95 \%$ confidence interval); $\mathrm{n}=10$. D Mean serum concentration-time profiles of MIC-1 following oral administration of once daily dose of idasanutlin for 5 days; data for last dose on day 5 (dose escalation, biomarker, and apoptosis cohort). Arithmetic means. $\mathrm{AUC}_{0-12 \mathrm{~h}}$, area under curve from 0 to $12 \mathrm{~h} ; \mathrm{C}_{\max }$, maximum plasma concentration; $\mathrm{QD}$, once daily; $\mathrm{QW}$, once weekly

for $>100$ days. The 2 patients with the longest duration of $\mathrm{SD}$ (> 600 days) were on the QD $\times 5$ schedule; both had sarcomas (Online Resource Fig. S1). One of these patients, who received $200 \mathrm{mg}$ idasanutlin $\mathrm{QD} \times 5$ and had a non-functional (deleterious) TP53 R158H mutant leiomyosarcoma, achieved SD for 620 days. The other patient, who received $800 \mathrm{mg}$ idasanutlin QD $\times 5$ and had a TP53 wild-type extraskeletal myxoid chondrosarcoma, achieved SD for 696 days.

\section{Pharmacokinetics}

Idasanutlin peak concentrations typically occurred 6 to $8 \mathrm{~h}$ after oral administration without food, and declined thereafter, with terminal half-life of $\approx 30 \mathrm{~h}$ (Fig. 2A). Exposure was approximately dose proportional after the first dose (i.e., day 1) and following repeat dosing (i.e., day 3 for $\mathrm{QD} \times 3$ regimens, day 5 for $\mathrm{QD} \times 5$ regimens), although increases appeared to be less than dose proportional at doses above $600 \mathrm{mg}$, suggesting a saturation in intestinal absorption at this dose level (Fig. 2B). However, interpatient variability in exposure was high with all dosing regimens (Fig. 2B). Exposure was approximately twofold higher on the final day of $\mathrm{QD} \times 3$ and $\mathrm{QD} \times 5$ dosing compared with the first dose (Fig. 2B), but there was no accumulation with $\mathrm{QW} \times 3$ dosing (data not shown). For a specified daily dose, cumulative idasanutlin exposure over the whole 28-day dosing cycle was greatest with a $\mathrm{QD} \times 5$ regimen, reflecting the higher total dose administered (i.e., 5 days of dosing vs. 3 days of dosing or 3 single doses).

\section{Food effects on idasanutlin pharmacokinetics}

Ten patients received $800-\mathrm{mg}$ doses of idasanutlin, either with a high-fat/high-calorie meal or while fasted. Dosing employed a half-replicate, crossover design, which resulted in 15 pairs of fed versus fasted data from the 10 patients. On average, idasanutlin exposure was higher when taken with food (mean maximum plasma concentration was $14 \%$ higher and area under the curve extrapolated to infinity was $43 \%$ higher), but variability was high and as the $90 \%$ confidence intervals encompassed unity, it was concluded that food had no clinically meaningful effect on idasanutlin exposure (Online Resource Table S7; Fig. 2C).

\section{Pharmacodynamic analysis}

After idasanutlin dosing, circulating macrophage inhibitory cytokine 1 (MIC-1) levels increased generally in a doseexposure-dependent manner (Fig. 2D). Consequently, trends in MIC-1 responses to treatment mirrored trends in idasanutlin exposure, as described in Population PK/PD Analysis section.

Sixteen of the 31 patients $(51.6 \%$ ) evaluated by positron emission tomography analysis for changes in tumor proliferation rates with idasanutlin treatment achieved a partial proliferative response as their best percentage maximum standardized uptake value change from baseline during cycle 1, indicating a decrease of $\geq 25 \%$ (Online Resource Fig. S2).

\section{Population PK/PD analysis}

Simulations with the indirect PK/MIC-1 model (Online Resource Supplementary Methods) indicated that despite some high variability, the release of MIC-1 following idasanutlin treatment is concentration dependent; the higher the idasanutlin concentrations, the higher the release of MIC-1. Weekly dosing with idasanutlin resulted in lower maximum release but a more sustainable effect on MIC-1 over the 28-day treatment cycle compared with a daily regimen (for the same level of dose) (Fig. 3).

The risk of grade $\geq 3$ neutropenia and thrombocytopenia events was exposure dependent as shown in Fig. 4. The higher the exposure over 28 days, the higher the risk of occurrence of events. Due to its lower exposure, the weekly dosing is predicted to have lower risk of neutropenia or thrombocytopenia compared with a daily regimen for the same dose level.

\section{Discussion}

The MTD for weekly dosing of idasanutlin MBP was identified as $3200 \mathrm{mg}$, while for daily dosing, it was $500 \mathrm{mg}$ $(\mathrm{QD} \times 5$ dosing $)$ and $1000 \mathrm{mg}(\mathrm{QD} \times 3$ dosing $)$. DLTs were primarily hematologic and gastrointestinal for all schedules. The MTD for daily dosing was much lower than that for weekly dosing, and as a result, a larger proportion of patients were treated at doses close to or above the MTD on the daily dosing schedule. The dose-escalation pattern resulted in more-frequent treatment-related AEs or 


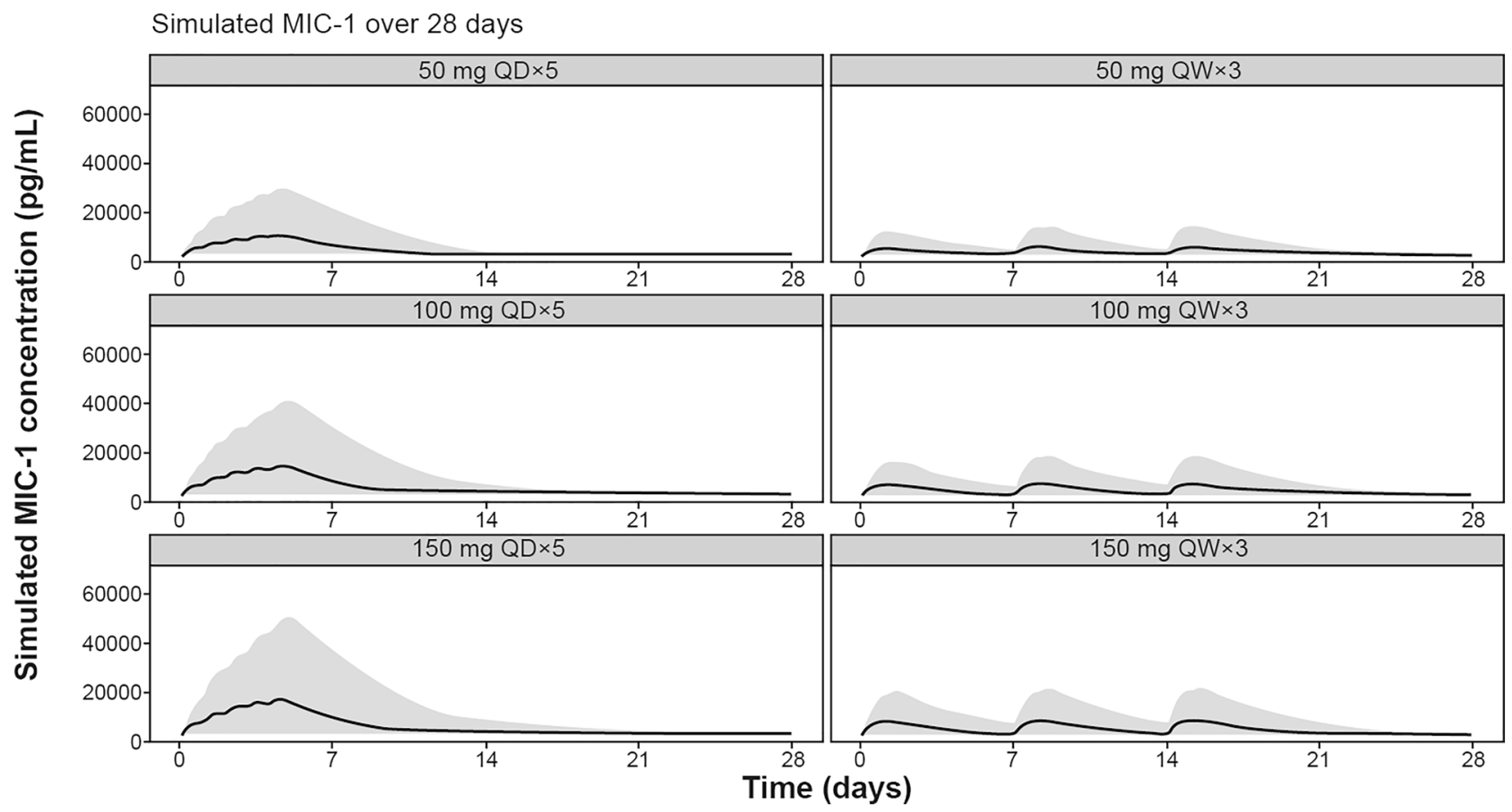

Fig. 3 Simulated MIC-1 release over 28 days following QD or QW regimen. The black line is the median of the predicted MIC-1 concentrations; the gray area is the $90 \%$ prediction interval around the

higher-intensity AEs, grade $\geq 3$ AEs, and SAEs with the daily schedule than with weekly dosing. The use of supportive therapies, including empiric anti-emetic prophylaxis and growth factor support, was able to manage the AEs.

The AE profile observed with idasanutlin was consistent with those in previous studies of MDM2 antagonists $[6,8]$. In solid tumors, hematologic effects must be considered after idasanutlin treatment, especially in combination

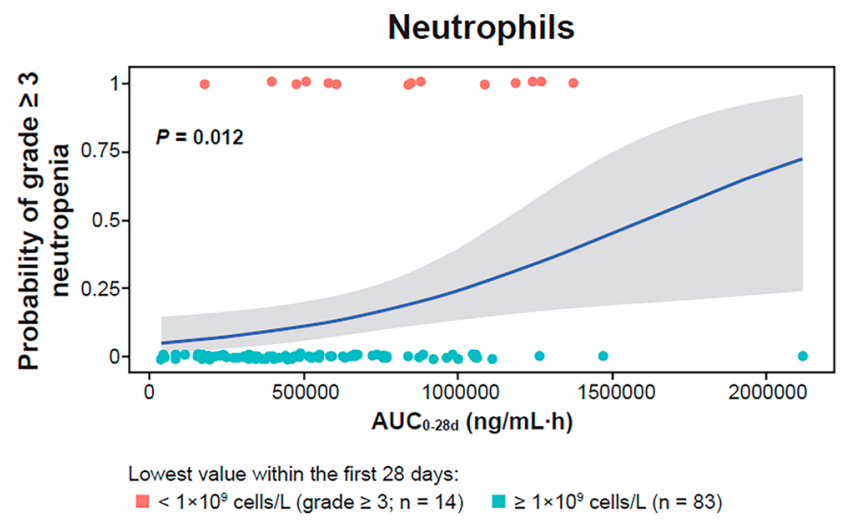

Fig. 4 Relationship between idasanutlin exposure and probability of occurrence of grade $\geq 3$ neutropenia and grade $\geq 3$ thrombocytopenia during cycle 1 . Idasanutlin exposure is the cumulative area under the curve over 28 days $\left(\mathrm{AUC}_{0-28 \mathrm{~d}}\right)$ in $\mathrm{ng} / \mathrm{mL} \cdot \mathrm{h}$ (individual exposures were derived using a population PK module developed median. Results are simulations with the indirect PK/MIC-1 model (stimulation of MIC-1 release). MIC-1, macrophage inhibitory cytokine 1; QD, once daily; QW, once weekly

therapy. Besides receiving supportive care for AE management (such as growth factor support to treat leukopenia and neutropenia, and transfusions of packed red blood cells to support anemia), patients must be monitored carefully for thrombocytopenia developing in the latter part of a dosing cycle or for neutropenia.

Idasanutlin's PK profile was characterized by high inter-patient variability, which confounded quantitative

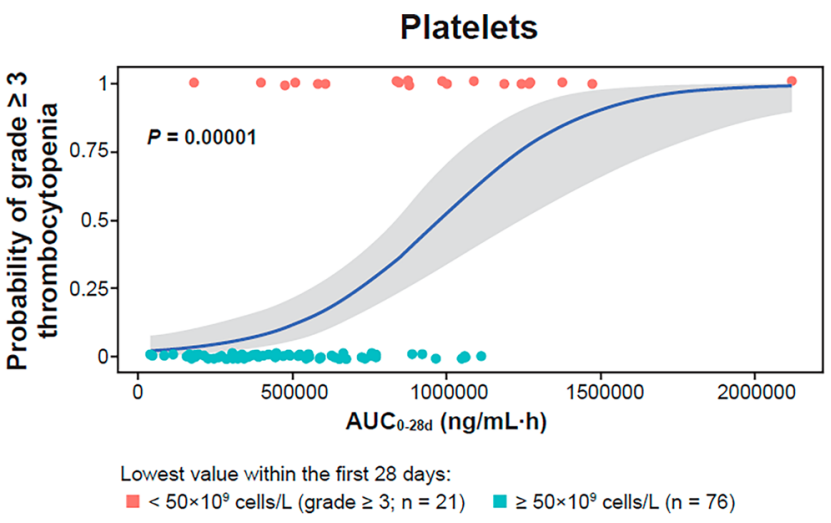

on data from different disease types [see Supplementary Methods]). Neutropenia grade $\geq 3$ events are the lowest absolute neutrophil count value $<1 \times 10^{9}$ cells/L with 28 days of treatment. Thrombocytopenia grade $\geq 3$ events are the lowest platelet count $<50 \times 10^{9}$ cells $/ L$ with 28 days of treatment 
comparisons between dosing regimens. Overall, cumulative idasanutlin exposure over the whole 28-day dosing cycle depended on the dosing regimen used and was greatest with a QD $\times 5$ regimen. The higher exposure was also reflected in a greater PD effect (Fig. 2D). No clinically meaningful food effect on idasanutlin exposure was observed. Consequently, induction of $\mathrm{p} 53$ as measured by MIC-1 levels occurred as a function of idasanutlin levels and treatment schedule, and trends in MIC-1 responses mirrored trends in idasanutlin exposure. MIC-1 induction was greater with daily than weekly dosing schedules, indicating that a daily schedule results in greater $\mathrm{p} 53$ activation than the weekly schedule.

PK/PD analyses also suggested an apparent relationship between idasanutlin exposure and hematologic toxicity. There was, however, no clearly identifiable difference in the idasanutlin exposure-hematological toxicity relationships between dosing regimens, suggesting that the apparent differences in tolerability between daily and weekly dosing can be attributed to differences in total idasanutlin exposure.

Population PK/PD modeling and simulations predict that, for the same dose level, a weekly dosing regimen will be associated with lower (as measured by MIC-1), although more sustained, target engagement over a 28-day treatment cycle and a lower risk of grade $\geq 3$ myelosuppressive events than a 5-day regimen. For the same cumulative dose, the PK/PD analyses did not suggest differentiation between the weekly and 5-day daily dosing regimens in target engagement or risk of grade $\geq 3$ myelosuppressive events. Exposure-response relationships based on monotherapy data predict limited target engagement and minimal risk of myelosuppression at idasanutlin doses currently being tested in combination studies.

Objective anti-tumor responses with idasanutlin (by RECIST 1.1 or Cheson criteria) were not observed in this study. Median durations of SD tended to be longer with the $\mathrm{QW} \times 3$ and $\mathrm{QD} \times 5$ schedules than with the $\mathrm{QD} \times 3$ schedule. Any associations of efficacy with TP53 mutation status or any specific mutations in TP53 cannot be made in the absence of objective clinical responses. The MTD on the daily dosing schedule for patients with solid tumors $(500 \mathrm{mg}$ for $\mathrm{QD} \times 5$ or $1000 \mathrm{mg}$ for $\mathrm{QD} \times 3$ ) is significantly lower than the 600-mg BID dose in the MBP formulation used in patients with relapsed/refractory acute myeloid leukemia in whom hematologic effects are a desired outcome [17]. It is reasonable to hypothesize that in patients with solid tumors, a dose higher than the MTD on either daily dosing schedule is required for monotherapy efficacy, or that combination therapy will need to be explored.

A comparison of MIC-1 elevation between the idasanutlin dosing schedules demonstrated that the daily schedule resulted in greater p53 activation than the weekly schedule. The $\mathrm{QD} \times 3$ regimen, however, did not achieve steady-state exposure and did not avert thrombocytopenia. Therefore, the $\mathrm{QD} \times 5$ schedule was selected for subsequent clinical trials based on the supportive evidence of p53 activation, comparable safety profiles of the $\mathrm{QD} \times 3$ and $\mathrm{QD} \times 5$ regimens, and ability to manage AEs with supportive therapies.

\section{Conclusion}

Idasanutlin led to exposure-dependent p53 activation with durable disease stabilization in select patients; no clinically meaningful food effect was observed. The daily $\mathrm{QD} \times 5$ schedule was identified for further clinical study, although alternative dosing schedules may be considered depending on the toxicity profile and/or combination partner. Idasanutlin is now being administered in an optimized spray dried powder formulation with approximately twofold higher bioavailability compared with the MBP formulation.

Supplementary information The online version contains supplementary material available at https://doi.org/10.1007/s10637-021-01141-2.

Acknowledgments We thank the patients and their families who participated in the study, and all the investigators and their staff. The authors thank Monica Reckner, Lori Jukofsky, and Steven Middleton for their contributions to the study. This study was supported by F. HoffmannLa Roche Ltd/Genentech Inc, a member of the Roche Group. Medical writing assistance was provided by Kia C. E. Walcott, PhD, and Jeff Frimpter, MPH, of Health Interactions, and funded by F. Hoffmann-La Roche Ltd.

Authors' contributions Concept and design: Yung-Jue Bang, Gwen L. Nichols, Lin Chi Chen, Lillian L. Siu.

Collection and assembly of data: Antoine Italiano, Wilson $\mathrm{H}$. Miller, Jr., Jean-Yves Blay, Jourik A. Gietema, Yung-Jue Bang, Linda R. Mileshkin, Brian Higgins, Gwen L. Nichols, Lin Chi Chen, Claire Petry, Candice Jamois, Lillian L. Siu.

Data analysis and interpretation: Antoine Italiano, Wilson H. Miller, Jr., Jean-Yves Blay, Jourik A. Gietema, Yung-Jue Bang, Linda R. Mileshkin, Hal W. Hirte, Brian Higgins, Steven Blotner, Gwen L. Nichols, Lin Chi Chen, Claire Petry, Qi Joy Yang, Christophe Schmitt, Candice Jamois, Lillian L. Siu.

Provision of study material or patients: Wilson H. Miller, Jr., JeanYves Blay, Yung-Jue Bang, Jourik A. Gietema, Linda R. Mileshkin, Hal W. Hirte, Lillian L. Siu.

Manuscript writing and final approval of manuscript: Antoine Italiano, Wilson H. Miller, Jr., Jean-Yves Blay, Jourik A. Gietema, Yung-Jue Bang, Linda R. Mileshkin, Hal W. Hirte, Brian Higgins, Steven Blotner, Gwen L. Nichols, Lin Chi Chen, Claire Petry, Qi Joy Yang, Christophe Schmitt, Candice Jamois, Lillian L. Siu.

Funding This study was sponsored by F. Hoffmann-La Roche, Ltd. The study sponsor participated in the concept, design, conduct, analysis and interpretation of the study and results, and in the writing of this report.

Data Availability Qualified researchers may request access to individual patient level data through the clinical study data request platform (https://vivli.org/). Further details on Roche's criteria for eligible studies are available here (https://vivli.org/members/ourmembers/). For further details on Roche's Global Policy on the Sharing of Clinical Information and how to request access to related clinical study documents, see here (https://www.roche.com/research_and_development/who_we_are_ how_we_work/clinical_trials/our_commitment_to_data_sharing.htm). 


\section{Declarations}

Ethics approval This study was conducted at eight participating institutions in accordance with the Declaration of Helsinki and Good Clinical Practice guidelines, after approval by a local human investigations committee and in accordance with an assurance filed with and approved by the Department of Health and Human Services, where appropriate. The study was registered on October 31, 2011, at ClinicalTrials.gov: NCT01462175.

Consent to participate All patients provided written informed consent.

Consent to publish All authors have approved the final version of the submitted work and agree to be accountable for all aspects of the report.

Conflicts of interests All authors received support from F. HoffmannLa Roche Ltd during the conduct of the study. Editorial support, provided by an independent medical writer and under the guidance of the authors, was funded by the sponsor. Antoine Italiano reports personal fees for consultancy from AstraZeneca, Novartis, Roche/Genentech, MSD, Bayer, Daiichi-Sankyo, and Astellas (outside the submitted work) and grants (to institution to conduct clinical trials) from AstraZeneca, Novartis, Roche/Genentech, MSD, Merck Serano, Bayer, and Bristol Myers Squibb (outside the submitted work); Wilson H. Miller, Jr. reports personal fees from Merck, Bristol Myers Squibb, Roche/Genentech, Novartis, Amgen, and GlaxoSmithKline (outside the submitted work); Jean-Yves Blay reports research support and honoraria from Roche, Novartis, and Amgen; Jourik A. Gietema reports research grants paid to the institution from Siemens and Abbvie; Yung-Jue Bang reports personal fees for consultancy from AstraZeneca, Novartis, Genentech/Roche, MSD, Merck Serano, Bayer, Bristol Myers Squibb, Eli Lilly, Taiho, Daiich-Sankyo, Astellas, BeiGene, GreenCross, Samyang Biopharm, Hanmi, and Genexine (outside the submitted work), grants (to institution to conduct clinical trials) from AstraZeneca, Novartis, Roche/Genentech, MSD, Merck Serano, Bayer, Bristol Myers Squibb, GlaxoSmithKline, Pfizer, Eli Lilly, Boeringer-Ingelheim, MacroGenics, Boston Biomedical, FivePrime, Curis, Taiho, Takeda, Ono, Daiichi Sankyo, Astellas, BeiGene, Green Cross, CKD Pharma, and Genexine (outside the submitted work); Linda R. Mileshkin has nothing to report; Hal W. Hirte has nothing to report; Brian Higgins reports employment from Roche/Genentech; Steven Blotner reports employment and stocks from Roche/Genentech; Gwen L. Nichols reports employment with Roche/Genentech during conduct of the study; Lin Chi Chen reports employment and equity and stock ownership from Roche/Genentech (during the conduct of the study); Claire Petry reports employment and stock ownership from Roche/Genentech; Qi Joy Yang reports employment with Certara (Canada); Christophe Schmitt reports employment and stock ownership from Roche/Genentech; Candice Jamois reports employment and stock ownership from Roche/ Genentech; Lillian L. Siu reports grants and non-financial support from Roche/Genentech (during the conduct of the study), personal fees from Roche/Genentech, Merck, Pfizer, AstraZeneca/Medimmune, Morphosys, Oncorus, Symphogen, Seattle Genetics, GlaxoSmithKline, Voronoi, Arvinas, Relay, Rubius, Janpix, Navire, and Novartis (outside the submitted work), grants (to institution to conduct clinical trials) from Bristol Myers Squibb, Pfizer, Boeringer-Ingelheim, GlaxoSmithKline, AstraZeneca, Merck, Astellas, Bayer, Abbvie, Amgen, Symphogen, Intensity Therapeutics, Mirati, Shattucks, and Avid (outside the submitted work), and that spouse is co-founder of Treadwell Therapeutics and has stock ownership from Agios.

Open Access This article is licensed under a Creative Commons Attribution 4.0 International License, which permits use, sharing, adaptation, distribution and reproduction in any medium or format, as long as you give appropriate credit to the original author(s) and the source, provide a link to the Creative Commons licence, and indicate if changes were made. The images or other third party material in this article are included in the article's Creative Commons licence, unless indicated otherwise in a credit line to the material. If material is not included in the article's Creative Commons licence and your intended use is not permitted by statutory regulation or exceeds the permitted use, you will need to obtain permission directly from the copyright holder. To view a copy of this licence, visit http://creativecommons.org/licenses/by/4.0/.

\section{References}

1. Vogelstein B, Lane D, Levine AJ (2000) Surfing the p53 network. Nature 408(6810):307. https://doi.org/10.1038/35042675

2. Konopleva M, Martinelli G, Daver N, Papayannidis C, Wei A, Higgins B, Ott M, Mascarenhas J, Andreeff M (2020) MDM2 inhibition: an important step forward in cancer therapy. Leukemia 34(11):2858-2874. https://doi.org/10.1038/s41375-020-0949-z

3. Vassilev LT, Vu BT, Graves B, Carvajal D, Podlaski F, Filipovic Z, Kong N, Kammlott U, Lukacs C, Klein C, Fotouhi N, Liu EA (2004) In vivo activation of the p53 pathway by small-molecule antagonists of MDM2. Science 303(5659):844. https://doi.org/10. 1126/science. 1092472

4. Ding Q, Zhang Z, Liu JJ, Jiang N, Zhang J, Ross TM, Chu XJ, Bartkovitz D, Podlaski F, Janson C, Tovar C, Filipovic ZM, Higgins B, Glenn K, Packman K, Vassilev LT, Graves B (2013) Discovery of RG7388, a potent and selective p53-MDM2 inhibitor in clinical development. J Med Chem 56(14):5979. https:// doi.org/10.1021/jm400487c

5. Tovar C, Rosinski J, Filipovic Z, Higgins B, Kolinsky K, Hilton H, Zhao X, Vu BT, Qing W, Packman K, Myklebost O, Heimbrook DC, Vassilev LT (2006) Small-molecule MDM2 antagonists reveal aberrant p53 signaling in cancer: implications for therapy. Proc Natl Acad Sci U S A 103(6):1888-1893. https://doi.org/10. 1073/pnas.0507493103

6. Ray-Coquard I, Blay JY, Italiano A, Le Cesne A, Penel N, Zhi J, Heil F, Rueger R, Graves B, Ding M, Geho D, Middleton SA, Vassilev LT, Nichols GL, Bui BN (2012) Effect of the MDM2 antagonist RG7112 on the P53 pathway in patients with MDM2amplified, well-differentiated or dedifferentiated liposarcoma: an exploratory proof-of-mechanism study. Lancet Oncol 13(11):1133. https://doi.org/10.1016/S1470-2045(12)70474-6

7. Obrador-Hevia A, Martinez-Font E, Felipe-Abrio I, CalabuigFariñas S, Serra-Sitjar M, López-Guerrero JA, Ramos R, Alemany R, Martín-Broto J (2015) RG7112, a small-molecule inhibitor of MDM2, enhances trabectedin response in soft tissue sarcomas. Cancer Invest 33(9):440-450. https://doi.org/10.3109/07357907. 2015.1064534

8. Andreeff M, Kelly KR, Yee K, Assouline S, Strair R, Popplewell L, Bowen D, Martinelli G, Drummond MW, Vyas P, Kirschbaum M, Iyer SP, Ruvolo V, Gonzalez GM, Huang X, Chen G, Graves B, Blotner S, Bridge P, Jukofsky L, Middleton S, Reckner M, Rueger R, Zhi J, Nichols G, Kojima K (2016) Results of the Phase I trial of RG7112, a small-molecule MDM2 antagonist in leukemia. Clin Cancer Res 22(4):868. https://doi.org/10.1158/1078-0432.CCR-15-0481

9. Tovar C, Graves B, Packman K, Filipovic Z, Higgins B, Xia M, Tardell C, Garrido R, Lee E, Kolinsky K, To KH, Linn M, Podlaski F, Wovkulich P, Vu B, Vassilev LT (2013) MDM2 small-molecule antagonist RG7112 activates p53 signaling and regresses human tumors in preclinical cancer models. Cancer Res 73(8):2587. https://doi.org/10.1158/0008-5472.CAN-12-2807

10. Higgins B, Tovar T, Glenn K, Walz A, Filipovic Z, Zhang YU, Dangl M, Lee E, Graves B, Vassilev L, Packman K (2013) Antitumor activity of the MDM2 antagonist RG7388. 
AACR-NCI-EORTC International Conference on Molecular Targets and Cancer Therapeutics annual meeting 12(suppl 11):B55

11. Higgins B, Glenn K, Walz A, Tovar C, Filipovic Z, Hussain S, Lee E, Kolinsky K, Tannu S, Adames V, Garrido R, Linn M, Meille C, Heimbrook D, Vassilev L, Packman K (2014) Preclinical optimization of MDM2 antagonist scheduling for cancer treatment by using a model-based approach. Clinical Cancer Res 20(14):3742. https://doi.org/10.1158/1078-0432.CCR-14-0460

12. Lakoma A, Barbieri E, Agarwal S, Jackson J, Chen Z, Kim Y, McVay M, Shohet JM, Kim ES (2015) The MDM2 smallmolecule inhibitor RG7388 leads to potent tumor inhibition in p53 wild-type neuroblastoma. Cell Death Discov 1. https://doi. org/10.1038/cddiscovery.2015.26

13. Phelps D, Bondra K, Seum S, Chronowski C, Leasure J, Kurmasheva RT, Middleton S, Wang D, Mo X, Houghton PJ (2015) Inhibition of MDM2 by RG7388 confers hypersensitivity to X-radiation in xenograft models of childhood sarcoma. Pediatr Blood Cancer 62(8):13451352. https://doi.org/10.1002/pbc.25465

14. Chen L, Rousseau RF, Middleton SA, Nichols GL, Newell DR, Lunec J, Tweddle DA (2015) Pre-clinical evaluation of the MDM2-p53 antagonist RG7388 alone and in combination with chemotherapy in neuroblastoma. Oncotarget 6(12):10207-10221. https://doi.org/10.18632/oncotarget.3504
15. Herting F, Herter S, Friess T, Muth G, Bacac M, Sulcova J, Umana P, Dangl M, Klein C (2016) Antitumour activity of the glycoengineered type II anti-CD20 antibody obinutuzumab (GA101) in combination with the MDM2-selective antagonist idasanutlin (RG7388). Eur J Haematol 97(5):461-470. https://doi.org/10. 1111/ejh.12756

16. O'Quigley J, Pepe M, Fisher L (1990) Continual reassessment method: a practical design for phase 1 clinical trials in cancer. Biometrics 46(1):33-48

17. Yee K, Papayannidis C, Vey N, Dickinson MJ, Kelly KR, Assouline S, Kasner M, Seiter K, Drummond MW, Yoon SS, Lee JH, Blotner S, Jukofsky L, Pierceall WE, Zhi J, Simon S, Higgins B, Nichols G, Monnet A, Muehlbauer S, Ott M, Chen LC, Martinelli G (2020) Murine double minute 2 inhibition alone or with cytarabine in acute myeloid leukemia: results from an idasanutlin phase 1/1b study $\star$. Leuk Res 100:106489. https://doi. org/10.1016/j.leukres.2020.106489

Publisher's Note Springer Nature remains neutral with regard to jurisdictional claims in published maps and institutional affiliations. 